\title{
Editorial
}

\section{Molecular Physics and Plasmas in Hypersonics}

\author{
Mario Capitelli ${ }^{*}, 1,2, \S$ and Annarita Laricchiuta ${ }^{2, \S}$ \\ ${ }^{I}$ Dipartimento di Chimica, Università degli Studi di Bari, Via Orabona 4, 70125 Bari, Italy \\ ${ }^{2}$ IMIP CNR, Via Amendola 122/D, 70125 Bari, Italy
}

In the aerospace scientific community, conventional macroscopic chemical-physical models are routinely used in CFD simulations of hypersonic flows. These models assume thermal (if not chemical) equilibrium on the belief that nonequilibrium effects play a minor role in every realistic case of interest but also to the requirement of computational efficiency. Non-equilibrium effects (vibrationally and electronically excited states, free electrons) have been considered by developing multi-temperature approaches, however under strong non-equilibrium conditions temperature is not a significant quantity to describe the distribution of particles over internal energy levels. Moreover the concept of temperature hides all the non equilibrium effects present in the tails of the relevant distributions which play an important role in promoting chemical reactions. Attempts to use state-to-state (StS) kinetics for the study of hypersonic flows are being considered by an increasing number of international groups and preliminary results are indeed encouraging. A strong effort however is required to transform these preliminary studies into a predictive tool.

This Special Issue is intended to give an up-to-date overview of the recent advances made, in the framework of the Phys4Entry EU project, in the simulation of hypersonic flows, from fundamental issues, as the molecular dynamics of elementary processes, to the development of state-to-state and new-concept macroscopic kinetic models to be included in detailed computational fluid dynamic codes for the rebuilding of experiments. Contributions focus also on more applicative subjects of relevance to aerospace engineering, such as (re)entry into planetary atmospheres and experimental methods in high-enthalpy wind tunnels, considering the physical phenomenology relative to the coupling among fluid dynamics, plasmas and electromagnetism.

Starting from the characterization of elementary processes, the challenging objective is the creation of a consistent state-selected cross section databases for the description of the elementary processes relevant to the

*Address correspondence to this author at the Dipartimento di Chimica, Università degli Studi di Bari, Via Orabona 4, 70125 Bari, Italy;

Tel: +39 0805929500; Fax: +39 0805929520;

E-mail: mario.capitelli@ba.imip.cnr.it

${ }^{\S}$ Guest Editors modeling of planetary atmospheres in the dissociation and ionization regimes, thus including electron-impact induced, heavy particle collision and heterogeneous processes. Quantum, semi- and quasi-classical approaches are exploited for the derivation of complete sets of cross sections resolved on internal (ro-vibrational and electronic) degrees of freedom moving to the treatment of triatomic molecular targets, with special attention to $\mathrm{CO}_{2}$ molecule.

Dynamical data are of paramount importance in the description of the non-equilibrium state-to-state kinetics and advanced models are presented that self-consistently coupled the kinetic equations with the Boltzmann equation for free electrons energy distribution function. However the highcomputational cost required by the full vibrational StS approach actually stimulated the renewed interest in newconcept macroscopic approaches, integrating the information of a detailed kinetics in different strategies, as global rate coefficients in non-equilibrium thermodynamics or a twolevel distribution model, hybridizing a multi-temperature model with a kinetic equation for the last vibrational level, or finally a multi-internal temperature model grouping levels in order to associate a temperature to different groups and to give an accurate description of the vibrational distribution. Furthermore the kinetic theory methods for the modeling of reacting flows are used for the simulation of the gas dynamic parameters and the heat transfer to the surface in a boundary layer near spacecraft non-catalytic surface entering the Mars atmosphere, accounting for the complexity of the kinetic model when the non-equilibrium $\mathrm{CO}_{2}$ internal level distributions are investigated with StS and quasi-stationary multi-temperature models.

Reliable estimates of radiative heat fluxes as well as of the radiation-induced changes in the fluid dynamic field around the vehicle are of paramount importance for the reduction of the uncertainty margin in the design of the thermal protection system. Again, conventional approaches are based on thermal equilibrium assumptions and on the optically thin plasma model that simplifies significantly the radiation transport model. On the one hand, StS models provide a consistent description of the radiation-hot gas interaction by modeling explicitly the excited state populations: synthetic emission spectra are therefore a direct output of the model to be compared with experimental measurements. The improvements in radiation models for the entry conditions in planetary atmosphere, with extended and accurate database of elementary radiative processes, are 
shown and an advanced collisional-radiative model for Jupiter atmosphere, that coupled self-consistently the kinetic equations for chemical processes, the Boltzmann equation for free electrons and the radiation transfer equation, is also presented. Modern problems of radiative aerothermodynamics of entering space vehicles, concerning the coupling of non-equilibrium processes with radiation heat transfer in shock layers are demonstrated and analyzed, giving a brief account on innovative magneto-hydrodynamic (MHD) technologies.

Again dealing with kinetic modeling, the significant progress achieved with Monte Carlo methods, i.e. PIC (Particle in Cell) and DSMC (Direct Simulation Monte Carlo), in the description of non-equilibrium in steep shock wave fronts is demonstrated, with numerical tools for CFD solvers able to correctly account of effects due to plasmamatter interaction and to rarefaction.

The ultimate example, integrating the concept of state-tostate models, deals with accurate CFD simulations in the design of hypersonic vehicles, allowing the estimation of error bars and nominal values of the design parameters and emphasizing the potential advantages of the detailed kinetic modules in the code validation with wind tunnel test cases. Moreover the relative importance of the implementation of the computationally expensive state-kinetic (non equilibrium) transport coefficients in CFD simulations of flow conditions is quantified.
New experimental tests are reported, explicitly designed to address the non-equilibrium character of plasmas generated in four different systems, including the post-shock air plasma in high-enthalpy shock tube, the shock wave generated by an acoustic shock tube, the subsonic nitrogen plasma jet produced by DC-Plasmatron facility and supersonic air plasma jets produced by Plasmatron facility.

The papers collected in this Special Issue offer a comprehensive view of the advances recently made in the investigation of different aspects connected to the predictive simulation of hypersonic flows in aerospace applications, emphasizing the role of non-equilibrium effects and the importance of StS approaches and representing a roadmap for the future of the research in this field.

As Guest Editors we would like to express our sincere appreciation to all invited authors. The relevant papers have been inspired by the invited topical talks presented during the $53^{\text {rd }}$ Course "Molecular Physics and Plasmas in Hypersonics" of the International School of Quantum Electronics of the Ettore Majorana Centre, held in Erice, Sicily (Italy) on September 8-15 2012. We wish to thank the European Office of Aerospace Research and Development (EOARD) Air Force Office of Scientific Research - United States Air Force Research Laboratory and the Italian Centre for Aerospace Research (CIRA) for their contribution to the success of the $53^{\text {rd }}$ Course.

(C) Capitelli and Laricchiuta; Licensee Bentham Open.

This is an open access article licensed under the terms of the Creative Commons Attribution Non-Commercial License (http://creativecommons.org/licenses/by-nc/3.0/) which permits unrestricted, non-commercial use, distribution and reproduction in any medium, provided the work is properly cited. 